Hautarzt 2021 · 72:692-699

https://doi.org/10.1007/s00105-021-04864-8

Angenommen: 23. Juni 2021

Online publiziert: 6 . Juli 2021

(c) Springer Medizin Verlag GmbH, ein Teil von Springer Nature 2021

\title{
Hidradenitis suppurativa/Acne inversa - operative Optionen, Rekonstruktionen und Kombi- nationen mit medikamentösen Therapien - Ein Update
}

\author{
P. Cramer ${ }^{1} \cdot$ S. Schneider-Burrus ${ }^{2} \cdot$ M. Kovács ${ }^{3} \cdot$ L. Scholl $^{1} \cdot$ M. Podda ${ }^{3} \cdot$ F. G. Bechara ${ }^{1}$ \\ ${ }^{1}$ Klinik für Dermatologie, Venerologie und Allergologie, Katholisches Klinikum Bochum, St. Josef-Hospital, \\ Klinik der Ruhr-Universität Bochum, Bochum, Deutschland \\ ${ }^{2}$ Zentrum für Dermatochirurgie, Havelklinik Berlin, Berlin, Deutschland \\ ${ }^{3}$ Hautklinik Klinikum Darmstadt - Akademisches Lehrkrankenhaus der Goethe-Universität Frankfurt am \\ Main, Darmstadt, Deutschland
}

Zusammenfassung

Hidradenitis suppurativa/Acne inversa (HS/AI) ist eine chronisch-entzündliche Hauterkrankung, deren Behandlung sowohl konservative als auch chirurgische Behandlungsmöglichkeiten umfasst. In den Hurley-Stadien II und III ist die chirurgische Resektion irreversibel zerstörten Gewebes anzustreben. Hierzu existieren verschiedene Resektionstechniken, die sich vor allem in ihrer Invasivität und Rezidivneigung unterscheiden. Bis heute gibt es keinen allgemein akzeptierten Konsens hinsichtlich verschiedener Resektions- und Rekonstruktionstechniken sowie der Einbeziehung medikamentöser Therapien in das therapeutische Gesamtkonzept.

\section{Schlüsselwörter}

Rekonstruktive chirurgische Verfahren · Immunmodulation · Wundheilung · Unterdruckwundtherapie · Rezidivrate

\section{Einleitung}

Hidradenitis suppurativa bzw. Acne inversa (HS/Al) ist eine chronisch-progrediente, entzündliche Hauterkrankung, die mit der Ausbildung von entzündlichen Knötchen, Abszessen und Fisteln, vorwiegend in intertriginösen Hautarealen, einhergeht [1-5]. Die konservative und chirurgische Behandlung richtet sich nach dem klinischen Schweregrad. Für die Indikationen zur chirurgischen Therapie hat die Stadieneinteilung nach Hurley, bei der 3 Schweregrade unterschieden werden, nach wie vor große Bedeutung [6].

Im vorliegenden Beitrag werden wir auf den aktuellen Stand der operativen Behandlungsoptionen bei HS/Al eingehen. Hierbei betrachten wir sowohl unterschiedliche Resektionstechniken als auch rekonstruktive Verfahren. Abschließend wird ein Überblick über die Integration von medikamentösen und chirurgischen Behandlungsansätzen gegeben, sowie die Versorgung während der COVID-19 Pandemie diskutiert.

\section{Chirurgische Optionen}

\section{Wann besteht die Indikation zu einer operativen Therapie?}

Eine Indikation zur Operation bei der HS/AI besteht immer dann, wenn irreversible Veränderungen der Hautstruktur vorliegen. Unter diesen harten Indikationen sind Fisteln, schwere Gewebedestruktionen, kontrahierte oder ziehende Narben und klinisch nicht auszuschließende maligne Prozesse zu verstehen, da diese nicht durch eine medikamentöse Therapie ausreichend therapiert werden können [7-9]. 

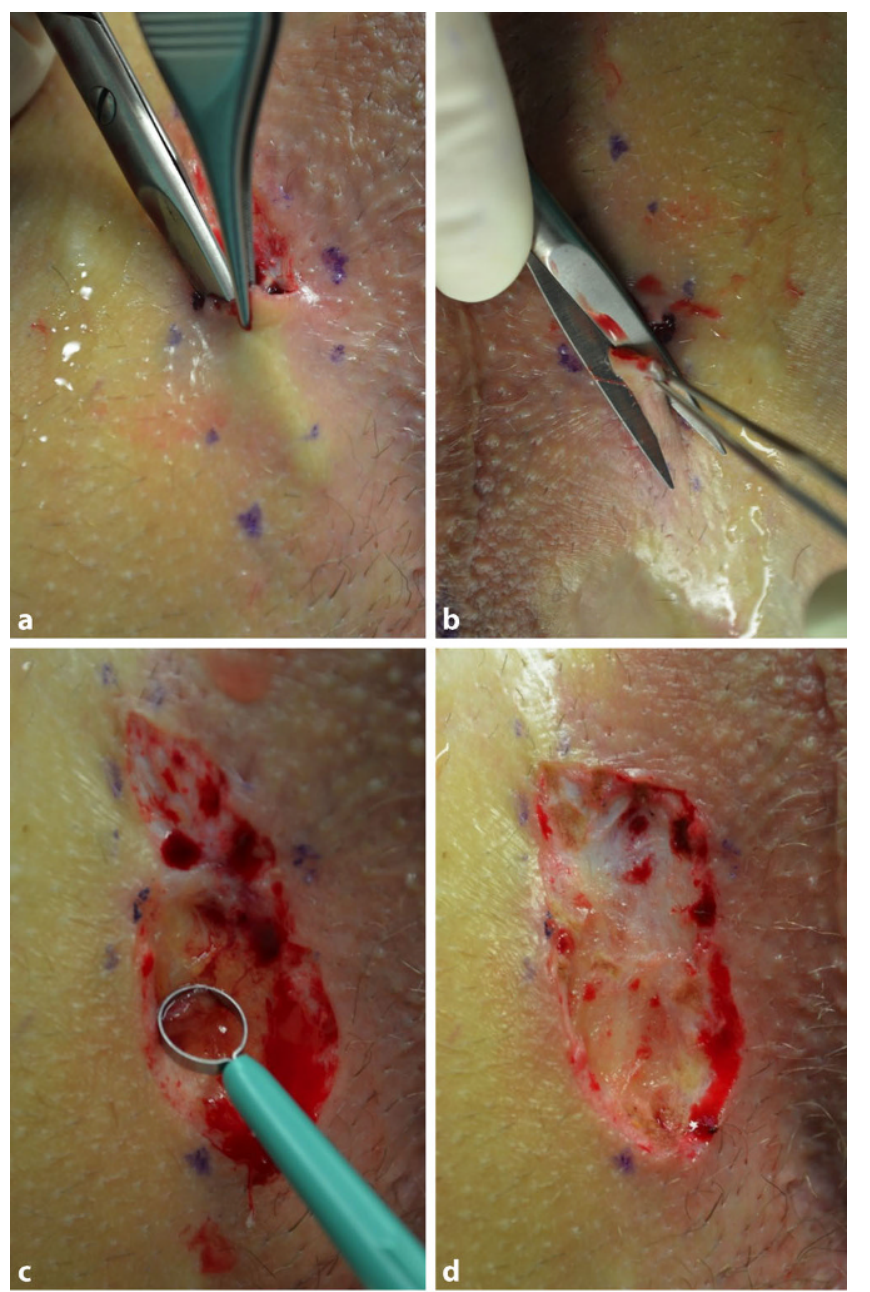

Abb. $1<$ Beispielhafte Darstellung der DeroofingTechnik bei einem oberflächlichen Sinustrakt. a Sondierung des Sinus nach Inzision mittels Schere oder Sonde, $\mathbf{b}$ Abtragung des Sinusdaches. c Am Boden des Sinus zeigt sich eine granulationsgewebeartige Schicht, diese wird mittels Kürette oder scharfem Löffel ausgekratzt. d Nach Kürettage zeigt sich am Grund des Sinus fibrotisches Gewebe. e Alternativ kann das Deroofing mittels Laser erfolgen, beispielhafte Darstellung mittels $\mathrm{CO}_{2}$ (Kohlenstoffdioxid)Laser

Des Weiteren ist zu erwähnen, dass im Hurley Stadium II und III die Operation den einzigen lokal kurativen Ansatz darstellt $[10,11]$. Aber auch im Hurley Stadium I und II können umschriebene Exzisionen ein wichtiger Pfeiler der Therapie sein.

\section{Welche operativen Optionen gibt es?}

\section{Inzision und Drainage}

Bei solitären akuten entzündlichen Knote/Abszessen sind Inzision und Drainage ein essenzieller Bestandteil der Therapie, um eine sofortige Schmerzreduktion zu erzielen.

Aufgrund des sehr hohen Rezidivrisikos, welches bei bis zu $100 \%$ liegt, sollten Inzision und Drainage jedoch immer in ein chirurgisch-medizinisches Gesamtkonzept eingebettet werden [12-14].

\section{Deroofing}

Die Dachentfernung eines Abszesses oder einer Fistel stellt einen Eingriff minimaler Invasivität im Hurley Stadium I oder || dar (-Abb. 1). Durch die Entfernung des "Daches" kann eine andauernde Entlastung erzielt werden, wobei so viel gesunde Haut wie möglich erhalten wird $[15,16]$. Zudem kann diese Methode auch in Kombination mit einer Sonde, der Elektrochirurgie oder mit einem $\mathrm{CO}_{2}$ (Kohlenstoffdioxid)-Laser verwendet werden $[15,17]$. Eine Kürettage sollte bei epithelisierten Böden durchgeführt werden, um das Rezidivrisiko zu senken. Die so entstandenen Defekte sollten sekundär heilen, was aufgrund der schellen Reepithelisierung der Defekte zu empfehlen ist $[18,19]$.

\section{STEEP}

Unter STEEP (,skin-tissue-sparing excision with electrosurgical peeling") versteht man die Exzision mit einer Kauterschlinge, mit der wiederholt tangentiale Abtragungen durchgeführt werden. Dieses Verfahren kommt zumeist im Hurley Stadium II und III zum Einsatz [20]. Wie beim Deroofing sollte auch hier eine sekundäre Wundheilung angestrebt werden [21].

In Bezug auf die Invasivität ist STEEP zwischen Deroofing und konventioneller Exzision einzuordnen.

\section{Exzision}

Die radikale Exzision wird in Bezug auf ihre Ausdehnung in der Literatur kontrovers diskutiert, auch wenn sie im Hurley Stadium II und III die einzige lokal kurative Behandlungsoption darstellt [22-25].

\section{॥In besonders komplexen Fällen ist eine bildgebende Diagnostik in Erwägung zu ziehen}

Diskutiert werden Methoden mit bis zu $2 \mathrm{~cm}$ Sicherheitsabstand oder auch die Exzision der gesamten behaarten Haut der Region ebenso wie die Exzision bis auf die Muskelfaszie oder aber die Begrenzung der Exzision auf die makroskopisch veränderten Hautschichten [26-29]. 

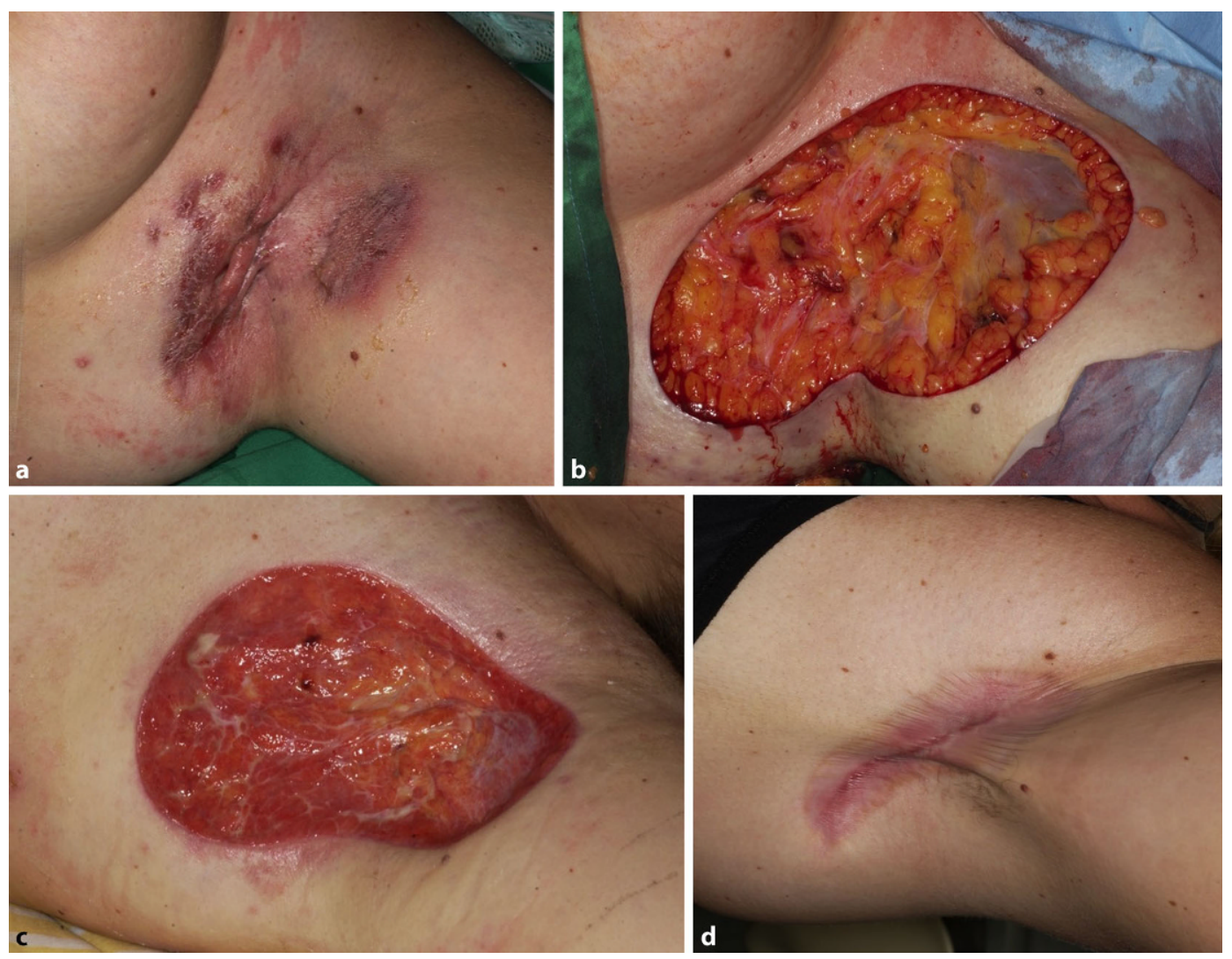

Abb. $2 \triangleleft$ Exzision einer Fistelplatte axillär links bei einer weiblichen Patientin mit Hidradenitis suppurativa/Acne inversa $(\mathrm{HS} / \mathrm{Al})$ im Hurley-Stadium III, sekundäre Wundheilung. a Präoperativer Befund axillar links, $\mathbf{b}$ Befund nach ausgedehnter Exzision der Fisteln, c Zustand nach sekundärer Wundheilung 4 Wochen postoperativ, $\mathbf{d}$ Ergebnis 6 Monate nach sekundärer Wundheilung

Bezüglich der Beurteilung der Resektionsränder werden die Anwendung von Methylenblau intraoperativ (Anfüllen der Fistelgänge) oder die präoperative Durchführung eines Jodwiderstandstests diskutiert [30-32].

Bildgebende Diagnostik wie eine MRT (Magnetresonanztomographie) oder eine Sonographie sind in besonders komplexen Fällen in Erwägung zu ziehen. Insbesondere perianal kann der Verlauf von Fisteln klinisch oder auch radiologisch häufig nicht eindeutig beurteilt werden. In diesen Fällen ist eine intraoperative Proktoskopie zum Ausschluss möglicher intraanaler oder rektaler Fistelöffnungen und somit zur Minimierung der Gefahr einer Schließmuskelverletzung dringend zu empfehlen [33].

Welche Resektionsverfahren werden aktuell empfohlen, und wie steht es um den Konsens?

In der Vergangenheit wurden zumeist radikale großflächige Exzisionen betroffener Hautregionen bevorzugt, während sich die aktuelle Entwicklung deutlich zu weniger invasiven und individueller abgestimmten operativen Vorgehensweisen bewegt. Es existiert jedoch nach wie vor kein einheitlicher Konsens über Art und Ausdehnung der chirurgischen Eingriffe.

》) Aktuell bewegt sich die Tendenz hin zu weniger invasiven, individueller abgestimmten

\section{Operationen}

Die Autoren empfehlen die vollständige Exzision des irreversibel zerstörten Gewebes bis auf das gesunde Fettgewebe, jedoch ohne die standardisierte Exzision der gesamten behaarten Haut einer anatomischen Region oder des gesunden Fettgewebes bis hinunter zu den Faszien.

\section{Rekonstruktionstechniken}

Zu den Verschlussmöglichkeiten der Operationswunden gehören neben der sekundären Wundheilung auch Dehnungsplastiken, ein- oder zweizeitige Spalthautdeckungen, lokale Lappenplastiken sowie fasziokutane, muskulokutane und freie Lappenplastiken. Welcher Verschluss gewählt wird, hängt vornehmlich von der
Größe und der Lokalisation des Defektes ab sowie von den Möglichkeiten der postoperativen Versorgung, besonders in Bezug auf Physiotherapie und Wundversorgung, aber auch von der Compliance des Patienten und nicht zuletzt von der Präferenz des Operateurs [34]. Einheitliche Empfehlungen fehlen in der aktuellen Literatur weiterhin.

\section{Sekundäre Wundheilung}

Den großen Vorteil dieser Methode stellt die sofortige Mobilisierung des Patienten, die akzeptable Narbenbildung, die schnelle Wiedereingliederung sowie das geringe Rezidiv- und Komplikationsrisiko dar [35-38]. Zu den Nachteilen gehören die starke Abhängigkeit der Wundheilung von der Tiefe und Größe der Wunde ebenso wie das Risiko von Kontrakturen [12, $39,40]$. Gerade im Inguinal-, Anogenitalund Abdominalbereich wird die sekundäre Wundheilung von viele Autoren trotz der aufwendigen postoperativen Wundversorgung und des hohen Bedarfs an physiotherapeutischer Betreuung bevorzugt [39, 41, 42]. Ein Beispiel für sekundäre Wund- 

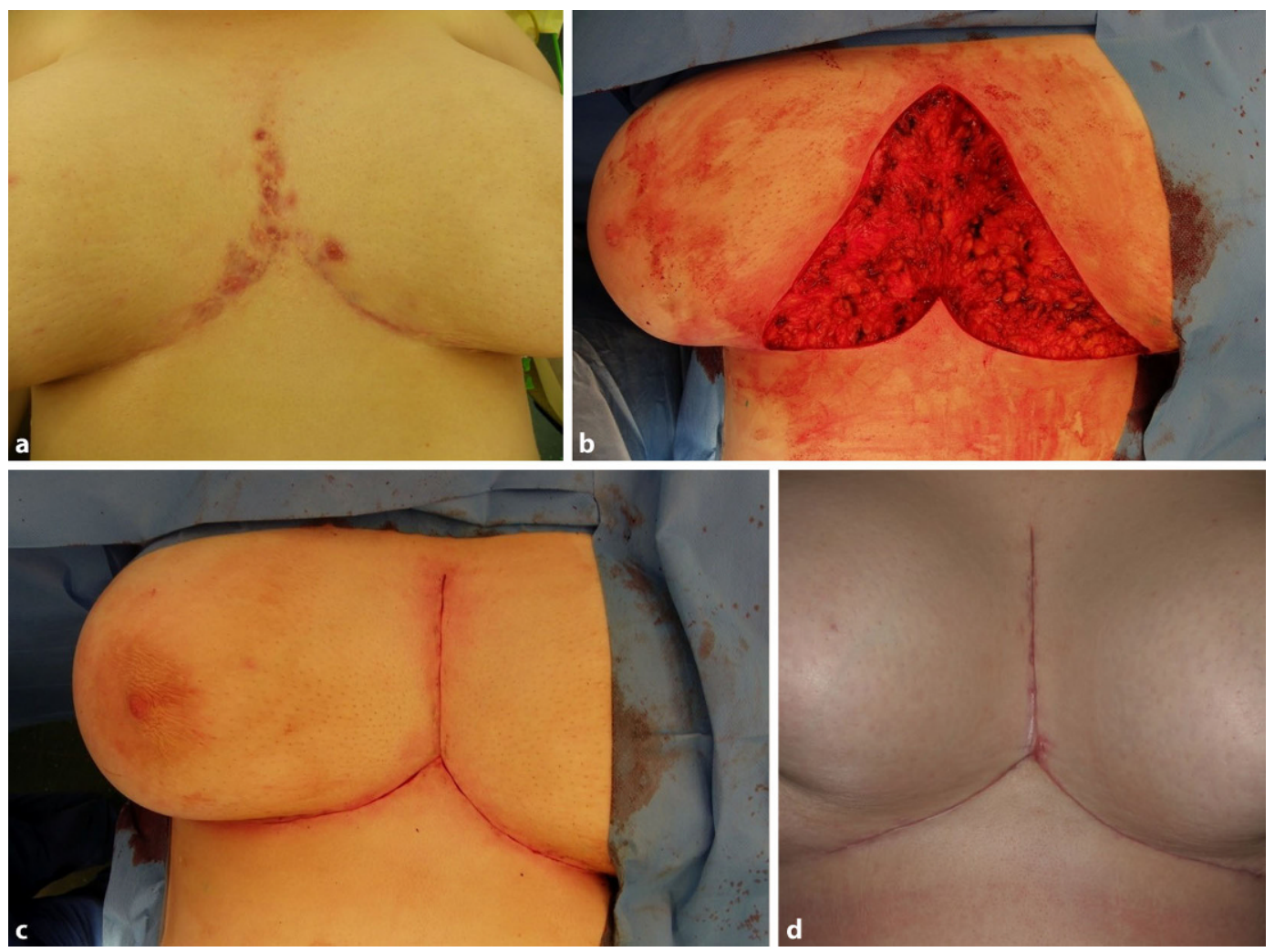

Abb. $3 \triangleleft$ Exzision mit primärem Wundverschluss be einer weiblichen Patientin mit Hidradenitis suppurativa/Acne inversa (HS/Al) im Hurley-Stadium II. a Präoperativer Befund sternal und inframammär, b intraoperativer Befund nach Exzision der Fistelgänge, c Zustand nach Rekonstruktion und primärem Wundverschluss mittels intrakutaner Naht, $\mathbf{d}$ Ergebnis 4 Monate nach primärem Wundverschluss heilung nach großflächiger Exzision einer Fistelplatte zeigt $\bullet$ Abb. 2.

\section{Dehnungsplastik}

Der primäre Verschluss mittels Dehnungsplastik kann bei umschriebenen Defekten nach lokaler Exzision bei Patienten mit Hurley-Stadium II sinnvoll sein, wobei aus Sicht der Autoren ein partieller Wundverschluss (z. B. partielle Dehnungsplastik) mit Einlage einer Lasche oder einer Drainage eine geeignete Methode darstellt, um die Heilung zu beschleunigen und das Kontrakturrisiko zu reduzieren [43]. Die relevanteste Limitation für den Einsatz der Dehnungsplastik ist die Größe der Läsion. Ein primärer Wundverschluss nach Resektion von Fisteln an der Brust ist beispielhaft gezeigt in - Abb. 3.

\section{Spalthauttransplantation}

Zumeist findet die Defektdeckung mittels einer Spalthauttransplantation im Rahmen eines zweizeitigen Operationskonzeptes, nach einer ausreichenden Wundkonditionierungsphase, ihre Anwendung, da sie meist das ästhetisch ansprechendere Ergebnis bietet (- Abb. 4; [41, 44, 45]). Oft kommt ein Meshgraft zum Einsatz, um mit einer möglichst kleinen Donorstelle einen möglichst großen Defekt decken zu können $[39,46]$. Grundsätzlich ist eine Spalthauttransplantation an allen Körperregionen möglich, wird aber nach umfangreichen Exzisionen im perianalen, glutealen, genitalen und inguinalen Bereich bevorzugt [47-49].

Eine hohe Compliance der Patienten ist ebenso essenziell wie die entsprechende Wundversorgung und eine ausreichende Narbenmassage und krankengymnastische Übungen. Aus Sicht der Autoren gehören der Skrotal- und der Penisbereich zu den typischen Lokalisationen der einzeitigen primären Spalthauttransplantation.

Die „reused skin graft technique" bietet den Vorteil der Einsparung der Donorstelle, da hierbei die Spalthaut mit einem Dermatom aus der resezierten erkrankten Haut entnommen und gemeshed wird [50].

Deutlich seltener wird die Nutzung von Dermisersatzpräparaten in der Literatur diskutiert, die zumeist mit einer Unterdruckwundtherapie (NPWT) für 10-20 Tage und anschließender Spalthauttransplantation kombiniert wird [51, 52].
Biosynthetischer Hautersatz bietet einekostenintensive Alternative, bei welcher jedoch die anschließende Spalthauttransplantation entfallen soll [53].

\section{NPWT}

Eine NPWT kommt besonders bei tiefen Defekten zur Beschleunigung der Wundgranulation und Reepithelisierung und zur Reduktion der Bakterienzahl und verbesserten Oxygenierung zum Einsatz [54].

Die Datenlage lässt weiterhin keine statisch basierte Empfehlung zum Einsatz der NPWT bei HS/Al zu.

\section{Lappenplastiken}

Zumeist kommen Lappenplastiken zur Rekonstruktion axillärer Defekte zum Einsatz, wenn größere Gefäße freiliegen oder wenn die Gefahr von Kontrakturen durch Spalthauttransplantate oder sekundäre Wundheilung als zu groß angesehen werden [55-57]. Hinzu kommt die kürzere Wundheilungsphase, ein ästhetisch ansprechendes Ergebnis sowie das Ausbleiben teils schmerzhafter Verbandswechsel [58-60]. 


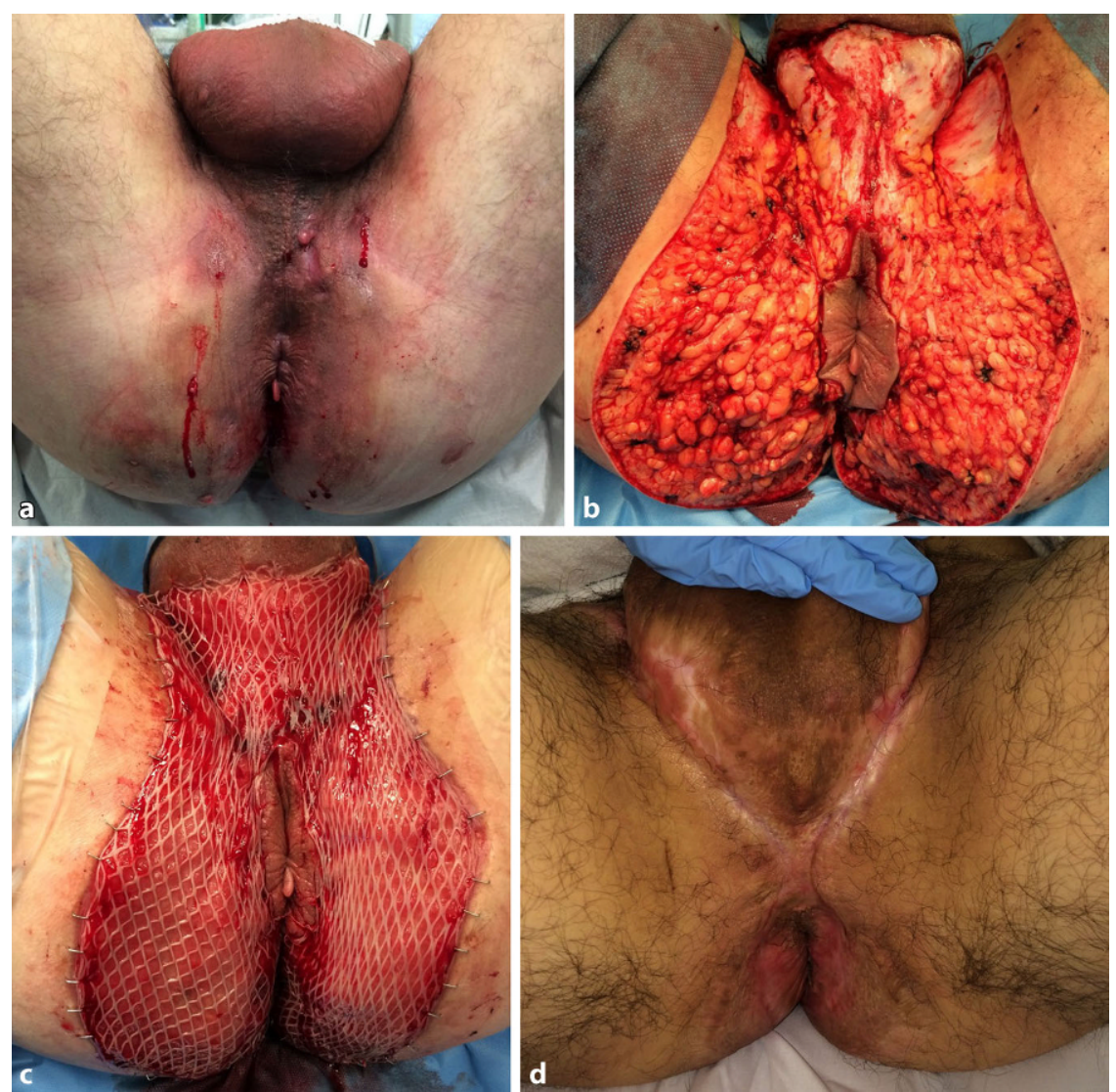

Abb. 4 ム Exzision anogenital bei einem männlichen Patienten mit Hidradenitis suppurativa/Acne inversa (HS/Al) im Hurley-Stadium III, Deckung mittels Spalthauttransplantation. a Präoperativer Befund nach Infiltration mit Tumeszenzanästhesielösung, b Befund am Ende der Operation nach ausgedehnter Exzision der Fistelplatten, c Zustand nach sekundärer Deckung mittels Spalthaut 4 Wochen nach Erstoperation, $\mathbf{d}$ Ergebnis 6 Monate nach Spalthauttransplantation

Welche Rekonstruktionsverfahren werden aktuell empfohlen, und wie steht es um den Konsens?

Aufgrund der weiterhin unzureichenden Datenlage kann aus evidenzbasierter Sicht keine einheitliche Empfehlung für eine bestimmte Rekonstruktionstechnik ausgesprochen werden.

Die Art des Verschlusses hängt nicht nur von der Größe und Lage des Defekts, sondern auch maßgeblich von der Compliance des Patienten, dem klinischen Bild und der Erfahrung des Operateurs ab und muss individuell entschieden werden. Insgesamt sollten Lappenplastiken zur Rekonstruktion bei der HS/Al eher zurückhaltend eingesetzt werden [61].
Welche Wundheilungszeiten sind zu erwarten?

Aufgrund sehr unterschiedlicher Definitionen sowie der sehr unterschiedlichen Rekonstruktionstechniken erstreckt sich der Wundheilungzeitraum in der Literatur von 2 Wochen bis zu 5 Monaten $[43,62]$. Nach Lappenplastiken wird sie zumeist mit 2-4 Wochen, nach Deroofing mit 2-6 Wochen und nach Spalthauttransplantation mit 2-8 Wochen angegeben [37, 47, 63]. Die längste Zeit bis zur vollständigen Wundheilung wird bei der sekundären Wundheilung mit einem Zeitraum von 3,5 Wochen bis zu 5 Monaten berichtet [41, $42,64]$.

\section{Rezidivrisiko}

Wie bereits zuvor oft berichtet, scheint das Ausmaß der Exzision die entscheidende Rolle für die Rezidivrate darzustellen, da oft davon ausgegangen wird, dass die Mehrzahl der Rezidive auf eine unvollständige Exzision zurückzuführen sind $[32,40$, 65].

Jedoch fehlen weiterhin einheitliche Daten in Bezug auf den Zusammenhang von Lokalisation, Exzisionstechnik und Verschlussmethode sowie eine einheitliche Definition des Begriffs „Rezidiv“.

\section{Wann ist die Kombination medikamentöser und operativer Verfahren indiziert?}

Bei der HS/Al handelt es sich um eine chronische, durch Entzündung und Gewebedestruktion gekennzeichnete Erkrankung. Bei Patienten, die ausschließlich entzündliche Läsionen zeigen, ist eine rein medikamentöse Therapie indiziert. Steht jedoch die Gewebedestruktion im Vordergrund, ist eine operative Therapie notwendig. Die meisten HS/Al-Patienten zeigen ein Mischbild aus Entzündung und Destruktion. Je nach Ausprägung dieser beiden Komponenten sollte entschieden werden, welche Therapie eingesetzt werden sollte. Bei Patienten mit schweren Entzündungen und einem Bedarf einer operativen Therapie sollte ein präoperatives Downstaging mit einer Antibiotikatherapie in Erwägung gezogen werden. Je nach Ausprägung und Therapieresistenz der Entzündung kann hierzu einer orale Kombinationsantibiotikatherapie bestehend aus Clindamycin und Rifampicin oder aber auch eine intravenöse Antibiotikatherapie mit Ertapenem in Erwägung gezogen werden. Dabei sollte die intravenöse Antibiotikatherapie mit Ertapenem den besonders komplizierten, therapierefraktären und ausgeprägten Hurley-III-Befunden vorbehalten bleiben, um mögliche Resistenzbildungen einzudämmen [66-70]. Leider fehlen weiterhin einheitliche Empfehlungen für das Downstaging hinsichtlich der Notwendigkeit, Dauer und Wahl der entzündungshemmenden Medikamente.

\section{॥ Auch für ein präoperatives Downstaging mit Antibiotika gibt es noch keine einheiltichen Empfehlungen}

Eine weitere Therapieoption ist die Kombination von Operation und Biologikathe- 
rapie. Studien konnten hier eine deutliche Überlegenheit der Kombination einer immunmodulatorischen Systemtherapie mit einer chirurgischen Therapie verglichen mit einer alleinigen immunmodulatorischen Systemtherapie, zeigen [71-88]. Es fehlen weiterhin klare Daten für eine Konsensbildung zur Kombination von Operation und biologischer Therapie.

\section{Eine neue Herausforderung: Versorgung von HS/AI-Patienten während der COVID-19-Pandemie}

Die COVID ("coronavirus disease")-19-Pandemie stellt seit über einem Jahr eine Herausforderung für das weltweite Gesundheitssystem dar. Hiervon betroffen ist auch die operative und medikamentöse Versorgung von HS/Al-Patienten.

Aktuell scheint es keinen Anhalt für schwerere Verläufen bei COVID-19-Infektionen bei HS/Al-Patienten bzw. bei Patienten unter einer Systemtherapie mit Adalimumab und Antibiotika zu geben. Trotzdem ist ein Therapiebeginn jeweils individuell und im Einzelfall zu prüfen. Die SARS-CoV2(,,severe acute respiratory syndrome coronavirus $2^{\prime \prime}$ )-Impfung wird für HS/AI-Patienten empfohlen, eine Therapie mit Adalimumab muss diesbezüglich nicht unterbrochen werden [89].

In einer deutschen Schwerpunktklinik für HS zeigte sich während der Pandemie in 2020 im Vergleich zu vor der Pandemie in 2019 eine Gesamtreduktion der behandelten Patienten um fast $40 \%$. Es wurden Patienten mit einem höheren Hurley-Grad behandelt, ebenso stieg während der Pandemie der prozentuale Anteil der Eingriffe in Vollnarkose, und es kam zu mehr postoperativen Nachblutungen. Somit schienen insbesondere Patienten mit einer hohen Krankheitslast/-aktivität die Klinik aufgesucht zu haben [90].

Ein weiterer Ansatz, der sich während Pandemiebedingungen herauskristallisiert hat, ist die Indikationserweiterung für Eingriffe in Tumeszenzlokalanästhesie. In dieser Technik können auch größere Areale mittels radikaler Exzision operiert werden. Hierdurch können anästhesiologische- und intensivmedizinische Ressourcen reduziert werden, die in anderen Abteilungen benötigt werden. Dieser An- satz sollte in der Post-COVID-19-Zeit weiter erwogen werden [91].

\section{Fazit für die Praxis}

- Da es an einheitlichen und validierten Daten fehlt, ist es derzeit nicht möglich, eine allgemein gültige Empfehlung bezüglich der Wahl bestimmter Resektions- und Rekonstruktionstechniken abzugeben.

- Im Rahmen der aktuellen Datenlage sind für die Wahl der Resektions- und Rektonstruktionsverfahren die Bedürfnisse des Patienten und die Erfahrung des Operateurs entscheidend.

- Die chirurgische Behandlung muss im Rahmen eines umfassenden Therapiekonzepts stehen, in welchem stets auch die Indikation zur medikamentösen Therapie geprüft werden sollte.

\section{Korrespondenzadresse}

Prof. Dr. F. G. Bechara

Klinik für Dermatologie, Venerologie und Allergologie, Katholisches Klinikum Bochum, St. Josef-Hospital, Klinik der Ruhr-Universität Bochum

Gudrunstr. 56, 44791 Bochum, Deutschland falk.bechara@klinikum-bochum.de bechara@web.de

\section{Einhaltung ethischer Richtlinien}

Interessenkonflikt. P. Cramer hat von der Firma Novartis und Incyte gesponserte Phase-II- und -IIIStudien betreut. S. Schneider-Burrus erhielt Reisekostenunterstützung oder Vergütung für Tätigkeiten im Rahmen klinischer Studien, Referenten- oder Beratertätigkeiten in alphabetischer Reihenfolge von folgenden Firmen: AbbVie Inc., AbbVie Deutschland $\mathrm{GmbH} \&$ Co. KG, Biogen GmbH Deutschland, Novartis Pharma GmbH, Roche Germany GmbH, UCB Pharma $\mathrm{GmbH}$, Deutschland. L. Scholl hat von der Firma Novar tis und Incyte gesponserte Phase-II- und -III-Studien betreut. F. G. Bechara: Referententätigkeit und Advisory Boards: AbbVie, Novartis, Janssen. Durchführung klinischer Studien: AbbVie, Novartis, Janssen, UCB, Incyte, Inflarx. M. Podda hat als Redner oder Berater von AbbVie, CSL, Boehringer Ingelheim, Eli Lilly, JanssenCilag, InflaRx, Novartis und UCB Honorare erhalten oder an klinischen Studien teilgenommen. M. Kovács hat als Redner oder Berater von AbbVie, Janssen-Cilag, UCB und Leo Pharma Honorare erhalten oder an klinischen Studien teilgenommen.

Für diesen Beitrag wurden von den Autoren keine Studien an Menschen oder Tieren durchgeführt. Für die aufgeführten Studien gelten die jeweils dort angegebenen ethischen Richtlinien.

\section{Literatur}

1. Kirschke J, Hessam S, Bechara FG (2015) Hidradenitis suppurativa/acne inversa: Ein Update.
Hautarzt 66:413-422. https://doi.org/10.1007/ s00105-015-3616-y

2. Sabat R (2017) Acne inversa/hidradenitis suppurativa: a challenge for the future. Hautarzt 68:994-998. https://doi.org/10.1007/s00105017-4079-0

3. Sabat R, Tsaousi A, Rossbacher J et al (2017) Acne inversa/hidradenitis suppurativa: an update. Hautarzt 68:999-1006. https://doi.org/10.1007/ s00105-017-4082-5

4. Patel ZS, Hoffman LK, Buse DC, Grinberg AS, Afifi L, Cohen SR et al (2017) Pain, psychological comorbidities, disability, and impaired quality of life in hidradenitis suppurativa. Curr Pain Headache Rep 21(12):49

5. McPhie ML, Bridgman AC, Kirchhof MG (2019) Combination therapies for hidradenitis suppurativa: a retrospective chart review of 31 patients. JCutan Med Surg 23(3):270-276

6. Hurley H (1989) Axillary hyperhidrosis, apocrine bromhidrosis, hidradenitis suppurativa and familial benign pemphigus. Surgical approach. In: Roenigk RK, Roenigk HH Jr (Hrsg) Dermatologic surgery. Principles and practice. Dekker, New York, S729-739

7. Bechara FG (2016) MIBHS: a concept to distinguish between medical and surgical treatment in hidradenitis suppurativa. Exp Dermatol 25(Suppl):8. https://doi.org/10.1111/exd.12954

8. Bechara FG, Hessam S (2016) Hidradenitis suppurativa. MMW Fortschr Med 158:52-53. https://doi.org/10.1007/s15006-016-8662-6

9. Alikhan A, Sayed C, Alavi A, Alhusayen R, Brassard A, Burkhart C et al (2019) North American clinical management guidelines for hidradenitis suppurativa: a publication from the United States and Canadian Hidradenitis Suppurativa Foundations: Part I: Diagnosis, evaluation, and the use of complementary and procedural management. J Am Acad Dermatol 81(1):76-90

10. Zouboulis CC, Bechara FG, Fritz K et al (2012) S1LeitliniezurTherapie der Hidradenitissuppurativa/ Acne inversa. J Dtsch Dermatol Ges 10(Suppl 5):S1-S31. https://doi.org/10.1111/j.1610-0387. 2012.08006.x

11. Zouboulis CC, Desai N, Emtestam L et al (2015) European S1 guideline for the treatment of hidradenitis suppurativa/acne inversa. J Eur Acad Dermatol Venereol 29:619-644. https://doi.org/ $10.1111 /$ jdv. 12966

12. Banerjee AK (1992) Surgical treatment of hidradenitis suppurativa. Br J Surg 79:863-866

13. Jemec GBE (2012) Clinical practice. Hidradenitis suppurativa. N Engl J Med 366:158-164. https:// doi.org/10.1056/NEJMcp1014163

14. Zouboulis CC, Bechara FG, Dickinson-BlokJL, GulliverW, Horváth B, Hughes Retal (2019) Hidradenitis suppurativa/acne inversa: a practical framework for treatment optimization-Systematic review and recommendations from the HS ALLIANCE working group. J Eur Acad Dermatol Venereol 33(1):19-31

15. van der Zee HH, Prens EP, Boer J (2010) Deroofing: a tissue-saving surgical technique for the treatment of mild to moderate hidradenitis suppurativa lesions. J Am Acad Dermatol 63:475-480. https:// doi.org/10.1016/j.jaad.2009.12.018

16. Dahmen RA, Gkalpakiotis S, Mardesicova L, Arenberger P, Arenbergerova M (2019) Deroofing followed by thorough sinus tract excision: a modified surgical approach for hidradenitis suppurativa. JDtsch Dermatol Ges 17(7):698-702

17. Aksakal AB, Adişen E (2008) Hidradenitis suppurativa: importance of early treatment; effi- 
cient treatment with electrosurgery. Dermatol Surg 34:228-231. https://doi.org/10.1111/j.15244725.2007.34042.x

18. van Hattem S, Spoo JR, Horváth B et al (2012) Surgical treatment of sinuses by deroofing in hidradenitis suppurativa. Dermatol Surg 38:494-497.https://doi.org/10.1111/j.1524-4725. 2011.02255.x

19. Hazen PG, Hazen BP (2010) Hidradenitis suppurativa: successful treatment using carbon dioxide laser excision and marsupialization. Dermatol Surg 36:208-213. https://doi.org/10.1111/j.15244725.2009.01427.x

20. Janse IC, Hellinga J, Blok JL et al (2016) Skin-tissuesparing excision with electrosurgical peeling: a case series in hidradenitis suppurativa. Acta Derm Venereol 96:390-391. https://doi.org/10.2340/ 00015555-2258

21. Blok JL, Spoo JR, Leeman FWJ et al (2015) Skin-Tissue-sparing Excision with Electrosurgical Peeling (STEEP): a surgical treatment option for severe hidradenitis suppurativa Hurley stage II/III. J Eur Acad Dermatol Venereol 29:379-382. https:// doi.org/10.1111/jdv. 12376

22. Endo Y, Tamura A, Ishikawa O, Miyachi Y (1998) Perianal hidradenitis suppurativa: early surgical treatment gives good results in chronic or recurrent cases. Br J Dermatol 139:906-910

23. Greeley PW (1951) Plastic surgical treatment of chronic suppurative hidradenitis. Plast Reconstr Surg 7:143-146

24. Mandal A, Watson J (2005) Experience with different treatment modules in hidradenitis suppuritiva: a study of 106 cases. Surgeon 3:23-26

25. Ingram JR, Collier F, Brown D, Burton T, Burton J, Chin MF et al (2019) British Association of Dermatologists guidelines for the management of hidradenitis suppurativa (acne inversa) 2018. Br J Dermatol 180(5):1009-1017

26. Bilali S, Todi V, Lila A et al (2012) Surgical treatment of chronic hidradenitis suppurativa in the gluteal and perianal regions. Acta Chir lugosI 59:91-95

27. Broadwater JR, Bryant RL, Petrino RA et al (1982) Advanced hidradenitis suppurativa. Review of surgical treatment in 23 patients. Am J Surg 144:668-670

28. Fernandes NC, Franco CPA, Lima CMO (2013) Hidradenitis suppurativa: retrospective study of 20 cases. An Bras Dermatol 88:480-481. https:// doi.org/10.1590/abd1806-4841.20131637

29. Martorell A, Giovanardi G, Gomez-Palencia P, Sanz-Motilva V (2019) Defining fistular patterns in hidradenitis suppurativa. Dermatol Surg 45(10):1237-1244

30. Humphries LS, Kueberuwa E, Beederman M, Gottlieb LJ (2016) Wide excision and healing by secondary intent for the surgical treatment of hidradenitis suppurativa: a single-center experience. J Plast Reconstr Aesthet Surg 69:554-566. https:// doi.org/10.1016/j.bjps.2015.12.004

31. Parks RW, Parks TG, Hughes LE(1997) Pathogenesis, clinical features and management of hidradenitis suppurativa. Ann R Coll Surg Engl 79:309-310

32. Rompel R, Petres $J(2000)$ Long-term results of wide surgical excision in 106 patients with hidradenitis suppurativa. Dermatol Surg 26:638-643

33. Takiyama H, Kazama S, Tanoue Y et al (2015) Efficacy of magnetic resonance imaging in the diagnosis of perianal hidradenitis suppurativa, complicated by anal fistulae: a report of two cases and review of the literature. Int J Surg Case Rep 15:107-111. https://doi.org/10.1016/j.ijscr.2015. 08.028
34. Scholl L, Hessam S, ReitenbachS, Bechara FG (2018) Operative Behandlungsoptionen bei Hidradenitis suppurativa/Acne inversa. Hautarzt 69:149-161. https://doi.org/10.1007/s00105-017-4114-1

35. Ariyan S, Krizek TJ (1976) Hidradenitis suppurativa of the groin, treated by excision and spontaneous healing. Plast Reconstr Surg 58:44-47

36. Ather S, Chan DSY, Leaper DJ, Harding KG (2006) Surgical treatment of hidradenitis suppurativa: case series and review of the literature. Int Wound J 3:159-169. https://doi.org/10.1111/j.1742-481X. 2006.00235.x

37. Balik E, Eren T, Bulut T et al (2009) Surgical approach to extensive hidradenitis suppurativa in the perineal/perianal and gluteal regions. World JSurg 33:481-487. https://doi.org/10.1007/ 500268-008-9845-9

38. Kagan RJ, Yakuboff KP, Warner P, Warden GD (2019) Surgical treatment of hidradenitis suppurativa: a 10-year experience. Surgery 138(4):734-741

39. Bieniek A, Matusiak L, Okulewicz-Gojlik D, Szepietowski JC (2010) Surgical treatment of hidradenitis suppurativa: experiences and recommendations. Dermatol Surg 36:1998-2004. https://doi.org/10. 1111/j.1524-4725.2010.01763.x

40. Harrison BJ, Mudge M, Hughes LE (1987) Recurrence after surgical treatment of hidradenitis suppurativa. Br Med J 294:487-489

41. Bocchini SF, Habr-Gama A, Kiss DR et al (2003) Gluteal and perianal hidradenitis suppurativa: surgical treatment by wide excision. Dis Colon Rectum 46:944-949. https://doi.org/10.1097/01. DCR.0000070046.16402.DF

42. Wollina U,TilpM, Meseg A etal (2012) Management of severe anogenital acne inversa (hidradenitis suppurativa). Dermatol Surg 38:110-117. https:// doi.org/10.1111/j.1524-4725.2011.02157.x

43. van Rappard DC, Mooij JE, Mekkes JR (2012) Mild to moderate hidradenitis suppurativa treated with local excision and primary closure. J Eur Acad Dermatol Venereol 26:898-902. https://doi.org/ 10.1111/j.1468-3083.2011.04203.x

44. Grösser A (1982) Surgical treatment of chronic axillary and genitocrural acne conglobata by splitthickness skin grafting. J Dermatol Surg Oncol 8:391-398. https://doi.org/10.1111/j.1524-4725. 1982.tb00303.x

45. Mustafa EB, Ali SD, Kurtz LH (1980) Hidradenitis suppurativa: review of the literature and management of the axillary lesion. J Natl Med Assoc 72:237-243

46. Watson JD (1985) Hidradenitis suppurativa-A clinical review. Br J Plast Surg 38:567-569. https:// doi.org/10.1016/0007-1226(85)90022-0

47. Anderson BB, Cadogan CA, Gangadharam D (1982) Hidradenitis suppurativa of the perineum, scrotum, and gluteal area: presentation, complications, and treatment. J Natl Med Assoc 74:999-1003

48. Chapman J (1972) The surgical treatment of hidradenitis suppurativa. J Natl Med Assoc 64:328-330

49. Grimstad $\emptyset$, Tzellos T, Dufour DN, Bremnes $\emptyset$, Skoie IM, Snekvik I et al (2019) Evaluation of medical and surgical treatments for hidradenitis suppurativa using real-life data from the Scandinavian registry (HISREG). J Eur Acad Dermatol Venereol 33(6):1164-1171

50. Kuo H, Ohara K(2003) Surgical treatment of chronic gluteal hidradenitis suppurativa: reused skin graft technique. Dermatol Surg 29:173-178. https://doi. org/10.1046/j.1524-4725.2003.29044.x

51. Gonzaga TA, Endorf FW, Mohr WJ, Ahrenholz DH (2013) Novel surgical approach for axillary hidradenitis suppurativa using a bilayer dermal regeneration template: a retrospective case study. JBurn Care Res 34:51-57. https://doi.org/10.1097/ BCR.0b013e31826a7be7

52. Rockwell WB, Yamashita Y, Hashimoto l et al (2014) Commentary: two-stage surgery for hidradenitis suppurativa: staged artificial dermis and skin grafting. Dermatol Surg 40:116-117. https://doi. org/10.1111/dsu. 12435

53. Melkun ET, Few JW (2005) The use of biosynthetic skin substitute (Biobrane) for axillary reconstruction after surgical excision for hidradenitis suppurativa. Plast Reconstr Surg 115:1385-1388. https:// doi.org/10.1097/01.PRS.0000157013.40191.91

54. Morykwas MJ, Argenta LC, Shelton-Brown El, McGuirt W (1997) Vacuum-assisted closure: a new method for wound control and treatment: animal studies and basic foundation. Ann Plast Surg 38:553-562

55. Büyükaşik $\mathrm{O}$, Hasdemir $\mathrm{AO}$, Kahramansoy $\mathrm{N}$ et al (2011) Surgical approach to extensive hidradenitis suppurativa. Dermatol Surg 37:835-842. https:// doi.org/10.1111/j.1524-4725.2011.01961.x

56. Civelek B, Aksoy K, Bilgen E et al (2010) Reconstructive options in severe cases of Hidradenitis suppurativa. Open Med. https://doi.org/10.2478/ s11536-009-0126-2

57. Rehman N, Kannan RY, Hassan S, Hart NB (2005) Thoracodorsal artery perforator (TAP) type I $\mathrm{V}-\mathrm{Y}$ advancement flap in axillary hidradenitis suppurativa. Br J Plast Surg 58:441-444. https:// doi.org/10.1016/j.bjps.2004.10.022

58. Altmann S, Fansa H, Schneider W (2001) Surgical treatment of axillary hidradenitis suppurativa. Chirurg 72:1413-1416

59. Rieger UM, Erba P, Pierer G, Kalbermatten DF (2009) Hidradenitis suppurativa of the groin treated by radical excision and defect closure by medial thigh lift: aesthetic surgery meets reconstructive surgery. J Plast Reconstr Aesthet Surg 62:1355-1360. https://doi.org/10.1016/j. bjps.2008.04.035

60. Soldin MG, Tulley P, Kaplan H et al (2000) Chronic axillary hidradenitis-the efficacy of wide excision and flap coverage. Br J Plast Surg 53:434-436. https://doi.org/10.1054/bjps.1999.3285

61. Janse I, Bieniek A, Horváth B, Matusiak L (2016) Surgical procedures in hidradenitis suppurativa. DermatolClin 34:97-109. https://doi.org/10.1016/ j.det.2015.08.007

62. Wiltz O, Schoetz DJ, Murray JJ et al (1990) Perianal hidradenitis suppurativa. The Lahey clinic experience. Dis Colon Rectum 33:731-734

63. Morgan WP, Harding KG, Hughes LE (1983) A comparison of skin grafting and healing by granulation, following axillary excision for hidradenitis suppurativa. Ann R Coll Surg Engl 65:235-236

64. Wollina U, Langner D, Heinig B, Nowak A (2017) Comorbidities, treatment, and outcome in severe anogenital inverse acne (hidradenitis suppurativa): a 15-year single center report. Int J Dermatol 56:109-115. https://doi.org/10.1111/ ijd.13393

65. Kagan RJ, Yakuboff KP, Warner P, Warden GD (2005) Surgical treatment of hidradenitis suppurativa: a 10-year experience. Surgery 138:734-740. https://doi.org/10.1016/j.surg.2005.06.053 ((discussion 740-741))

66. Bettoli V, Manfredini M, Massoli L, Carillo C, Barozzi A, Amendolagine G et al (2019) Rates of antibiotic resistance/sensitivity in bacterial cultures of hidradenitis suppurativa patients. J Eur Acad Dermatol Venereol 33(5):930-936 
67. Schneller-Pavelescu L, Vergara-de Caso E, Martorell A, Romaní J, Lázaro M, Vilarrasa E et al (2019) Interruption of oral clindamycin plus rifampicin therapy in patients with hidradenitis suppurativa: an observational study to assess prevalence and causes. J Am Acad Dermatol 80(5):1455-1457

68. Albrecht J, Baine PA, Ladizinski B, Jemec GB, Bigby M (2019) Long-term clinical safety of clindamycin and rifampicin combination for the treatment of hidradenitis suppurativa: a critically appraised topic. Br J Dermatol 180(4):749-755

69. Marasca C, Masarà A, Annunziata MC, Bettoli V, Luciano MA, Fabbrocini G (2019) Long-term clinical safety of clindamycin and rifampicin combination for the treatment of hidradenitis suppurativa: strategy to reduce side effects, improving patients' compliance. Br JDermatol 180(4):949

70. Delage $M$, Jais JP, Lam T, Guet-Revillet $H_{\text {, }}$ Ungeheuer MN, Consigny PHet al (2020) Rifampinmoxifloxacin-metronidazole combination therapy for severe Hurley stage 1 hidradenitis suppurativa: prospective short-term trial and one-year followup in 28 consecutive patients. J Am Acad Dermatol. https://doi.org/10.1016/j.jaad.2020.01.007

71. Andersen RK, Jemec GB (2017) Treatments for hidradenitis suppurativa. Clin Dermatol 35:218-224. https://doi.org/10.1016/j.clindermatol.2016.10. 018

72. Falola RA, DeFazio MV, Anghel EL et al (2016) What heals hidradenitis suppurativa: surgery, immunosuppression, or both? Plast Reconstr Surg 138:219S-229S. https://doi.org/10.1097/ PRS.0000000000002671

73. Van Rappard DC, Mekkes JR (2012) Treatment of severe hidradenitis suppurativa with infliximab in combination with surgical interventions. $\mathrm{Br} J$ Dermatol 167:206-208. https://doi.org/10.1111/j. 1365-2133.2012.10807.x

74. Alikhan A, Sayed C, Alavi A, Alhusayen R, Brassard A, Burkhart C et al (2019) North American clinical management guidelines for hidradenitis suppurativa: a publication from the United States and Canadian Hidradenitis Suppurativa Foundations: Part II: Topical, intralesional, and systemic medical management. J Am Acad Dermatol 81(1):91-101

75. Argyropoulou M, Kanni T, Kyprianou M, Melachroinopoulos N, Giamarellos-Bourboulis EJ (2019) Cost-savings of adalimumab in hidradenitis suppurativa: a retrospective analysis of a real-world cohort. Br JDermatol 180(5):1161-1168

76. Zouboulis CC, Okun MM, Prens EP, Gniadecki R, Foley PA, Lynde $C$ et al (2019) Long-term adalimumab efficacy in patients with moderateto-severe hidradenitis suppurativa/acne inversa: 3 -year results of a phase 3 open-label extension study. J Am Acad Dermatol 80(1):60-69.e2

77. Jemec GB, Okun MM, Forman SB, Gulliver WP Prens EP, Mrowietz U et al (2019) Adalimumab medium-term dosing strategy in moderate-tosevere hidradenitis suppurativa: integrated results from the phase 3, randomized, placebo-controlled, PIONEER trials. Br J Dermatol 181(5):967-975

78. Oskardmay AN, Miles JA, Sayed CJ (2019) Determining the optimal dose of infliximab for treatment of hidradenitis suppurativa. J Am Acad Dermatol 81(3):702-708

79. Vossen $A R$, van Doorn MB, van der Zee HH, PrensEP (2019) Apremilast for moderate hidradenitis suppurativa: results of a randomized controlled trial. J Am Acad Dermatol 80(1):80-88

80. Kerdel FR, Azevedo FA, Kerdel Don C, Don FA, Fabbrocini G, Kerdel FA (2019) Apremilast for the treatment of mild-to-moderate hidradenitis

\section{Hidradenitis suppurativa /acne inversa-surgical options, reconstruction and combinations with drug therapies-an update}

Hidradenitis suppurativa/acne inversa (HS/AI) is a chronic inflammatory skin disease whose treatment includes both conservative and surgical treatment options. In Hurley stages II and III, surgical resection of irreversibly destroyed tissue should be the objective. For this purpose several resection techniques exist, which differ primarily with regard to their invasiveness and tendency to recur. To date, there is no generally accepted consensus on the use of different resection and reconstruction techniques or the inclusion of drug therapies in the overall therapeutic concept.

\section{Keywords}

Reconstructive surgical procedures · Immunomodulation · Wound healing $\cdot$ Negative-pressure wound therapy - Recurrence rate

suppurativa in a prospective, open-label, phase 2 study. JDrugs Dermatol 18(2):170-176

81. André R, Marescassier H, Gabay C, Pittet B,

Laffitte $E$ (2019) Long-term therapy with anakinra in hidradenitis suppurativa in three patients. Int J Dermatol 58(11):e208-e209

82. Prussick L, Rothstein B, Joshipura D, Saraiya A, Turkowski Y, Abdat R et al (2019) Open-label, investigator-initiated, singlesite exploratory trial evaluating secukinumab, an anti-interleukin-17A monoclonal antibody, for patients with moderateto-severe hidradenitis suppurativa. Br J Dermatol 181(3):609-611

83. Casseres RG, Kahn JS, Her MJ, Rosmarin D (2019) Guselkumab in the treatment of hidradenitis suppurativa: a retrospective chart review. J Am Acad Dermatol 81(1):265-267

84. Scheinfeld N (2013) Hidradenitis suppurativa: a practical review of possible medical treatments based on over 350 hidradenitis patients. Dermatol Online J 19(4):1

85. Provini LE, Stellar JJ, Stetzer MN, Nguyen PD, Jen M (2019) Combination hyperbaric oxygen therapy and ustekinumab for severe hidradenitis suppurativa. Pediatr Dermatol 36(3):381-383

86. Kovacs M, Podda M (2019) Guselkumab in the treatment of severe hidradenitis suppurativa. JEur Acad Dermatol Venereol 33(3):e140-e141

87. Casseres RG, Prussick L, Zacanaro R, Rothstein B, Joshipura D et al (2020) Secukinumab in the treatment of moderate to severe hidradenitis suppurativa: results of an open label trial. J Am Acad Dermatol. https://doi.org/10.1016/j.jaad. 2020.02.005

88. Savage KT, Brant EG, Santillan MR, Morss PC, Salian P et al (2020) Methotrexate shows benefit in a subset of patients with severe hidradenitis suppurativa. Int J Womens Dermatol. https://doi. org/10.1016/j.ijwd.2020.02.007

89. Giamarellos-Bourboulis EJ, Bettoli V, Jemec GBE, del Marmol V, Marzano AV, Prens EP, Tzellos T, Zouboulis CC (2021)Anti-COVID-19measurements forhidradenitissuppurativa patients. ExpDermatol 30:18-22

90. Kovacs M, Podda M (2021) Current changes in the standard of care of hidradenitis suppurativa patients in a German specialist clinic due to the COVID-19 pandemic. Exp Dermatol 30:82

91. Podda M, Hasche E, Kovacs M (2021) Tumescence vs. general anesthesia: a precious alternative in large hidradenitis suppurativa surgery learned due to the COVID-19 pandemic. Exp Dermatol 30:70-71 